\title{
Valores de tensão na determinação da curva de retenção de água de solos do Cerrado
}

\author{
Euzebio Medrado da Silva(1), Jorge Enoch Furquim Werneck Lima(1), Juscelino Antônio de Azevedo(1) \\ e Lineu Neiva Rodrigues ${ }^{(1)}$
}

${ }^{(1)}$ Embrapa Cerrados, Caixa Postal 08223, CEP 73310-970 Planaltina, DF. E-mail: euzebio@cpac.embrapa.br, jorge@cpac.embrapa.br,
juscelin@cpac.embrapa.br, lineu@cpac.embrapa.br

Resumo - A curva de retenção de água do solo é essencial para o estudo das relações solo-água. A escolha do número e da combinação de pontos a serem levantados para o seu traçado normalmente é feita de forma arbitrária. O objetivo deste trabalho foi definir o menor número e a melhor combinação de pontos de tensão que resultem na descrição da curva de retenção de água de solos do Cerrado. Utilizaram-se cinco curvas de retenção de diferentes solos como referência, cada uma com 15 pontos medidos pelo método da centrífuga e ajustada ao modelo de Genuchten. A partir desses 15 pontos, 385 curvas foram geradas para cada solo, com seis, sete, oito e nove pontos, a fim de serem comparadas com a curva de referência. A análise da distribuição estatística da soma de quadrados dos erros padronizados, entre as curvas geradas e as respectivas curvas de referência, permitiu definir um modelo de probabilidade que serviu como instrumento para a escolha das melhores combinações de pontos de tensão. A combinação definida pelos oito valores de tensão de 1, 3, 6, 10, 35, 84, 611 e $1.515 \mathrm{kPa}$ é a recomendada para o levantamento das curvas de retenção de água de solos do Cerrado.

Termos para indexação: solo-água, centrifugação, pontos de tensão.

\section{Tension values to describe soil-water retention curve on Cerrado soils}

\begin{abstract}
Soil-water retention curve is essential in studying soil-water relations. The choice of the number and the combination of the tension points for describing soil-water retention curve is usually done in an arbitrary manner. The objective of this work was to define the least number and the best combination of the necessary tension points to describe the adequate soil-water retention curve of Cerrado soils. Five soil-water retention curves from different soils were utilized and taken as a reference, each one described with 15 points, measured by the centrifuge method and fitted with Genuchten model. For each soil sample, 385 curves were generated with six, seven, eight, and nine points, in order to be compared with the reference curve. The statistical distribution analysis of the sum of the normalized square error between the generated curves and the respective reference curve allowed defining a probability model that was used as a tool for choosing the best combinations of the tension points. The combination defined by the eight tension values of 1, 3, 6, 10, 35, 84, 611 e $1.515 \mathrm{kPa}$ is recommended for describing soil-water retention curve for Cerrado soils.
\end{abstract}

Index terms: soil-water, centrifugation, tension points.

\section{Introdução}

A determinação da curva de retenção de água do solo, que representa a relação entre o teor de água e a energia com a qual ela está retida, é essencial no estudo das relações solo-água. Sua determinação, efetuada por meio de técnicas tradicionais de laboratório, tais como a da câmara de pressão (Richards, 1965) e da centrífuga (Silva \& Azevedo, 2002), baseia-se no levantamento de certo número de pontos, normalmente selecionados de forma arbitrária. Com base nesses pontos, é traçada uma curva para representar as características de retenção da água do solo.

Vários modelos empíricos (Brooks \& Corey, 1966; Genuchten, 1980; Rossi \& Nimmo, 1994; Assouline et al., 1998; Kastanek \& Nielsen, 2001; Prunty \& Casey, 2002) têm sido propostos e empregados para a descrição da curva de retenção a partir de um determinado número de pontos medidos. Segundo Kastanek \& Nielsen (2001), as funções empíricas continuam sendo usadas para 
ajustar as curvas de retenção de água do solo porque ainda não foram desenvolvidas expressões matemáticas teóricas capazes de representar adequadamente essa relação físico-hídrica. Na tentativa de simplificar o processo de levantamento da curva de retenção, Silva et al. (1990) desenvolveram um método empírico para estimativa da curva de retenção e da quantidade de água disponível no solo, em função apenas da umidade na capacidade de campo para diferentes classes de textura de solos brasileiros.

No ajuste da curva de retenção, é previsível que, quanto maior o número de pontos, mais representativa será a curva de retenção; no entanto, mais oneroso será seu levantamento. Na seleção dos pontos para o traçado da curva de retenção, observa-se que não existe critério padronizado, sendo comum o uso de seis a oito pontos, incluindo o conteúdo de água do solo na saturação, para o ajuste do modelo matemático adotado, como pode ser constatado em vários trabalhos (Silva et al., 1990; Macedo et al., 1998; Spera et al., 2000; Silva \& Azevedo, 2002; Abreu et al., 2004; Cintra et al., 2004). A utilização arbitrária de um determinado conjunto de pontos, sem um critério de seleção estatístico definido, pode resultar em curvas de retenção de água não representativas do solo. Por isso, é fundamental o estudo que leve à definição do número mínimo e à melhor combinação de pontos, em bases estatísticas, que resulte na descrição adequada da curva de retenção da água do solo.

O objetivo deste trabalho foi definir o menor número e a melhor combinação de pontos de tensão que resulte na descrição da curva característica da água de solos do Cerrado.

\section{Material e Métodos}

Foram coletadas amostras de solos representativos do bioma Cerrado (Reatto et al., 1998), em cinco pontos amostrais, localizados na área experimental da Embrapa Cerrados, no Distrito Federal (Tabela 1). Em cada ponto amostral, foram coletadas quatro amostras indeformadas da camada de 15 a $20 \mathrm{~cm}$, utilizando cilindros metálicos de $5,1 \mathrm{~cm}$ de altura e $100 \mathrm{~cm}^{3}$ de volume, para a determinação da densidade do solo (Embrapa, 1997) e da curva de retenção de água do solo (Silva \& Azevedo, 2002). As características físicas das amostras dos solos estão apresentadas na Tabela 1. Dos locais amostrados, somente o de solo LVd era cultivado. Esse fato refletiu-se na densidade do solo relativamente elevada, de $1.207 \mathrm{~kg} \mathrm{~m}^{-3}$, quando comparada com sua densidade na condição natural, que se situa em torno de $900 \mathrm{~kg} \mathrm{~m}^{-3}$, conforme resultados de Spera et al. (2000), em solos de classe similar.

No laboratório, as amostras foram saturadas, por pelo menos 72 horas, com uma lâmina de água de aproximadamente $4 \mathrm{~cm}$ de altura, para avaliação de seu conteúdo volumétrico na saturação. Em seguida, cada conjunto de quatro amostras de um mesmo solo foi submetido somente ao processo da centrifugação, para a obtenção da curva média de retenção de cada local amostrado, aplicando-se os seguintes valores de tensão: 1, 3, 6, 10, $35,60,84,101,201,406,611,806,1.027,1.308$ e $1.515 \mathrm{kPa}$, utilizando o período de centrifugação de 2 horas (Silva \& Azevedo, 2002). As rotações correspondentes às tensões de 1 a $6 \mathrm{kPa}$ foram rigorosamente ajustadas na centrífuga por meio de um tacômetro eletrônico e as demais rotações foram estabelecidas utilizando os próprios dispositivos de controle da centrífuga. Os procedimentos de cálculo para a conversão de rotação em tensão e as características da centrífuga utilizada encontram-se descritos em Silva \& Azevedo (2001).

No ajuste dos 15 valores pontuais do conteúdo de água versus tensão, para cada solo, foi utilizado o modelo de Genuchten (1980), o qual contém os seguintes parâmetros: $\theta_{\mathrm{s}}, \theta_{\mathrm{r}}, \mathrm{n}, \alpha$. Nesse modelo, $\theta_{\mathrm{s}}$ define a assíntota superior da curva, representando o conteúdo saturado de água no solo, enquanto $\theta_{\mathrm{r}}$ define a assíntota inferior da curva, representando o conteúdo residual de água no solo; o parâmetro n é adimensional e reflete a forma da curva, ao tempo em que o $\alpha$ é expresso em $\mathrm{kPa}^{-1} \mathrm{e}$ é afetado pela escala da tensão da água no solo. Os parâmetros $\theta_{\mathrm{r}}$, n e $\alpha$ foram obtidos por meio da rotina Solver da planilha eletrônica Excel, ao passo que o parâmetro $\theta_{\mathrm{s}}$ foi determinado por meio da pesagem direta da amostra do solo saturado, antes de ser submetido ao processo de centrifugação, e, portanto, não foi objeto de ajuste. As curvas de retenção de água com 15 pontos serviram como referência para fins de comparação com as outras curvas de retenção, geradas com menor número de pontos.

A fim de estabelecer possíveis combinações (Tabela 2) para o traçado das curvas de retenção com menor número de pontos de tensão, primeiro, fixou-se como base $\mathrm{B}$ o conjunto dos cinco pontos com as menores tensões (1, 3, 6, 10 e $35 \mathrm{kPa})$. Adicionando-se ao conjunto $\mathrm{B}$, seqüencialmente, cada uma das outras tensões (de 60 a $1.515 \mathrm{kPa}$ ), foram obtidas dez alternativas distintas de curvas de retenção de seis pontos. Juntando-se um ponto de tensão ao conjunto B, 
para formar outro conjunto-base, agora com seis pontos - por exemplo, B com $60 \mathrm{kPa}$-, e combinando esse novo conjunto-base, seqüencialmente, com cada uma das tensões subseqüentes (de 84 a $1.515 \mathrm{kPa}$ ), foram geradas nove alternativas distintas de curvas de retenção com sete pontos. Tomando os demais conjuntos-base de seis pontos (de B84 a B1308) e combinando cada um deles com as respectivas tensões subseqüentes, foram obtidas mais 36 alternativas, totalizando, assim, 45 curvas distintas com sete pontos. Seguindo essa mesma sistemática para formação de curvas de retenção de oito pontos, foram obtidas 120 alternativas e assim também foi feito para obtenção das curvas com nove pontos, as quais resultaram em 210 alternativas. Desse modo, foi obtido um total de 385 alternativas de curvas de retenção para cada um dos solos. A análise das curvas de retenção, geradas com um número de pontos superior a nove, dependeu de os resultados indicarem ou não a necessidade de sua inclusão neste trabalho.

Para cada uma dessas 385 curvas de retenção, com seis, sete, oito e nove pontos, foram determinados, por meio de regressão não linear, usando a rotina Solver do Excel, os respectivos parâmetros $\theta_{\mathrm{r}}$, n e $\alpha$, do modelo de Genuchten (1980). Nesse ajuste, o valor de $\theta_{\mathrm{s}}$ foi comum nas 385 curvas de retenção de um mesmo solo.
Com o modelo ajustado de todas as combinações de pontos de tensão em cada solo, calculou-se, para cada caso, o conteúdo volumétrico de água correspondente a cada um dos 15 valores de tensão e determinou-se a soma de quadrados de suas respectivas diferenças (SQ $\left.{ }_{\text {erro}}\right)$, em relação ao conteúdo volumétrico de água calculado com o modelo de Genuchten (1980) ajustado com 15 pontos. Desse modo, foi possível comparar os 15 valores de conteúdo de água medidos da curva de retenção completa, com os respectivos valores de conteúdo de água calculados, de cada uma das 385 curvas de retenção incompletas, em cada solo, obtendo as respectivas somas dos quadrados das diferenças (SQerro).

Em cada solo, os 385 valores de SQerro foram analisados em forma de histograma de freqüências, com nove classes (Iman \& Conover, 1983), para verificar o tipo de função estatística que melhor representava a distribuição desses erros. A análise conjunta da distribuição estatística padronizada dos valores de $\mathrm{SQ}_{\text {erro }}$ dos solos estudados permitiu calcular a probabilidade de ocorrência desses erros, associada a cada combinação de pontos de tensão e seu respectivo valor médio entre solos. Com base nessa probabilidade média, foi então realizada a classificação das 385 alternativas de curva de retenção, permitindo, assim, selecionar, para cada número de pontos de tensão, a combinação

Tabela 1. Coordenadas geográficas dos locais amostrados e classes e características físicas dos solos ${ }^{(1)}$.

\begin{tabular}{|c|c|c|c|c|c|c|c|}
\hline \multirow[t]{2}{*}{ Latitude $\left({ }^{\circ}\right)$} & \multirow[t]{2}{*}{ Longitude $\left({ }^{\circ}\right)$} & \multirow[t]{2}{*}{ Classe de solo } & \multicolumn{5}{|c|}{ Características físicas dos solos } \\
\hline & & & $\overline{\text { Argila (\%) }}$ & Silte $(\%)$ & $\begin{array}{c}\text { Areia grossa } \\
(\%)\end{array}$ & $\begin{array}{c}\text { Areia fina } \\
(\%)\end{array}$ & $\begin{array}{l}\text { Densidade do } \\
\text { solo }\left(\mathrm{kg} \mathrm{m}^{-3}\right)\end{array}$ \\
\hline 15,61126 & 47,73068 & $\begin{array}{l}\text { Neossolo Quartzarênico distrófico } \\
\mathrm{A} \text { fraco relevo plano fase Cerrado Típico } \\
\left(\mathrm{RQ}_{1}\right)\end{array}$ & 5,20 & 0,40 & 10,00 & 84,40 & 1.311 \\
\hline 15,61272 & 47,73967 & $\begin{array}{l}\text { Neossolo Quartzarênico distrófico } \\
\text { A fraco relevo suave-ondulado fase } \\
\text { Cerrado Ralo }\left(\mathrm{RQ}_{2}\right)\end{array}$ & 11,20 & 0,40 & 12,00 & 76,40 & 1.296 \\
\hline 15,61160 & 47,74325 & $\begin{array}{l}\text { Latossolo Vermelho-Amarelo } \\
\text { distrófico A moderado textura média } \\
\text { relevo plano a suave-ondulado fase } \\
\text { Cerrado Típico }\left(\text { LVAd }_{1}\right)\end{array}$ & 32,80 & 0,30 & 14,20 & 52,70 & 1.317 \\
\hline 15,60780 & 47,74466 & $\begin{array}{l}\text { Latossolo Vermelho-Amarelo } \\
\text { distrófico A moderado textura argilosa } \\
\text { relevo plano a suave-ondulado fase } \\
\text { Cerrado Típico }\left(\text { LVAd }_{2}\right)\end{array}$ & 55,90 & 16,90 & 4,70 & 22,50 & 1.092 \\
\hline 15,59432 & 47,73213 & $\begin{array}{l}\text { Latossolo Vermelho distrófico } \\
\text { A moderado textura argilosa relevo } \\
\text { plano a suave-ondulado fase Cerrado } \\
\text { Típico (LVd) }\end{array}$ & 52,40 & 12,50 & 5,40 & 26,70 & 1.207 \\
\hline
\end{tabular}

${ }^{(1)}$ Latitude e longitude de acordo com Datum WGS 84 e classes de solos segundo Embrapa (1999). 
que resulta na curva de retenção mais próxima da curva descrita com 15 pontos, considerada como curva de referência neste trabalho. Adicionalmente, ao se aplicar o teste $\mathrm{t}$ de Student para comparação de médias (Neufeld, 2003), obteve-se o nível de significância das diferenças entre cada uma das melhores alternativas de combinação de seis, sete, oito e nove pontos.

\section{Resultados e Discussão}

Os parâmetros das curvas de retenção de água dos solos com 15 pontos de tensão, ajustadas ao modelo de Genuchten (1980), estão apresentados na Tabela 3. No processo de otimização não linear, estabeleceu-se que os valores dos parâmetros $\theta_{\mathrm{r}}$, n e $\alpha$ fossem maiores do que zero, não sendo necessário impor a restrição para

Tabela 2. Esquema da combinação de pontos de tensão para formar as alternativas de curvas de retenção com seis a nove pontos, a partir dos dados da curva de retenção completa de 15 pontos.

\begin{tabular}{|c|c|c|c|c|c|c|c|c|c|c|}
\hline \multicolumn{10}{|c|}{ Tensão da água no solo $(\mathrm{kPa})$} & \multirow[t]{2}{*}{$\mathrm{NP}^{(1)}$} \\
\hline 60 & 84 & 101 & 201 & 406 & 611 & 806 & 1027 & 1308 & 1515 & \\
\hline \multirow[t]{38}{*}{$\mathrm{B}^{(2)}$} & & & & & & & & & & 6 \\
\hline & ${ }^{(3)} \mathrm{B} 60$ & B84 & B101 & B201 & B406 & B611 & B806 & B1027 & B1308 & 7 \\
\hline & & $\mathrm{B} 60.84^{(4)}$ & B 60.101 & B 60.201 & B 60.406 & B60.611 & $\mathrm{B} 60.806$ & B 60.1027 & B 60.1308 & 8 \\
\hline & & & B84.101 & B84.201 & B84.406 & B84.611 & B84.806 & B84.1027 & B84.1308 & 8 \\
\hline & & & & $\mathrm{B} 101.201$ & $\mathrm{~B} 101.406$ & $\mathrm{~B} 101.611$ & $\mathrm{~B} 101.806$ & B101.1027 & $\mathrm{B} 101.1308$ & 8 \\
\hline & & & & & $\mathrm{B} 201.406$ & $\mathrm{~B} 201.611$ & $\mathrm{~B} 201.806$ & B201.1027 & B201.1308 & 8 \\
\hline & & & & & & B406.611 & $\mathrm{B} 406.806$ & B406.1027 & B406.1308 & 8 \\
\hline & & & & & & & $\mathrm{B} 611.806$ & B611.1027 & B611.1308 & 8 \\
\hline & & & & & & & & B806.1027 & B806.1308 & 8 \\
\hline & & & & & & & & & B1027.1308 & 8 \\
\hline & & & $\mathrm{B} 60.84 .101^{(5)}$ & B60.84.201 & B 60.84 .406 & B60.84.611 & B60.84.806 & B60.84.1027 & B 60.84 .1308 & 9 \\
\hline & & & & B60.101.201 & B 60.101 .406 & B60.101.611 & B 60.101 .806 & B60.101.1027 & B 60.101 .1308 & 9 \\
\hline & & & & & B 60.201 .406 & B 60.201 .611 & B 60.201 .806 & B60.201.1027 & B 60.201 .1308 & 9 \\
\hline & & & & & & B 60.406 .611 & B 60.406 .806 & B60.406.1027 & B 60.406 .1308 & 9 \\
\hline & & & & & & & B60.611.806 & B60.611.1027 & B60.611.1308 & 9 \\
\hline & & & & & & & & B60.806.1027 & B 60.806 .1308 & 9 \\
\hline & & & & & & & & & B 60.1027 .1308 & 9 \\
\hline & & & & B84.101.201 & B84.101.406 & B84.101.611 & B84.101.806 & B84.101.1027 & B84.101.1308 & 9 \\
\hline & & & & & B84.201.406 & B84.201.611 & B84.201.806 & B84.201.1027 & B84.201.1308 & 9 \\
\hline & & & & & & B84.406.611 & B84.406.806 & B84.406.1027 & B84.406.1308 & 9 \\
\hline & & & & & & & B84.611.806 & B84.611.1027 & B84.611.1308 & 9 \\
\hline & & & & & & & & B84.806.1027 & B84.806.1308 & 9 \\
\hline & & & & & & & & & B84.1027.1308 & 9 \\
\hline & & & & & B101.201.406 & B101.201.611 & B101.201.806 & B101.201.1027 & B101.201.1308 & 9 \\
\hline & & & & & & B101.406.611 & B101.406.806 & B101.406.1027 & B101.406.1308 & 9 \\
\hline & & & & & & & B101.611.806 & B101.611.1027 & B101.611.1308 & 9 \\
\hline & & & & & & & & B101.806.1027 & B101.806.1308 & 9 \\
\hline & & & & & & & & & B101.1027.1308 & 9 \\
\hline & & & & & & B201.406.611 & B201.406.806 & B201.406.1027 & B201.406.1308 & 9 \\
\hline & & & & & & & B201.611.806 & B201.611.1027 & B201.611.1308 & 9 \\
\hline & & & & & & & & B201.806.1027 & B201.806.1308 & 9 \\
\hline & & & & & & & & & B201.1027.1308 & 9 \\
\hline & & & & & & & B406.611.806 & B406.611.1027 & B406.611.1308 & 9 \\
\hline & & & & & & & & B406.806.1027 & B406.806.1308 & 9 \\
\hline & & & & & & & & & B406.1027.1308 & 9 \\
\hline & & & & & & & & B611.806.1027 & B611.806.1308 & 9 \\
\hline & & & & & & & & & B611.1027.1308 & 9 \\
\hline & & & & & & & & & B806.1027.1308 & 9 \\
\hline
\end{tabular}

(1)NP: número de pontos de tensão utilizados na curva de retenção considerada. (2)B: conjunto base das tensões de 1 , 3 , 6 , 10 e 35 kPa, que, associado aos valores de tensão subseqüentes de 60 a $1.515 \mathrm{kPa}$, forma dez combinações de curvas de retenção com seis pontos de tensão; por exemplo, a combinação de B com 84 forma o seguinte conjunto de seis pontos de tensão: 1, 3, 6, 10, 35 e 84 kPa. (3) B60: conjunto base das tensões de 1, 3, 6, 10, 35 e $60 \mathrm{kPa}$, que, associado aos valores de tensão subseqüentes de 84 a $1515 \mathrm{kPa}$, forma 45 combinações de curvas de retenção com sete pontos; por exemplo, a combinação de B60 com 84 forma o seguinte conjunto de sete pontos de tensão: 1, 3, 6, 10, 35, 60 e 84 kPa. (4)B60.84: conjunto base das tensões de 1, 3, 6, 10, 35, 60 e $84 \mathrm{kPa}$, que, associado aos valores de tensão subseqüentes de 101 a 1515 kPa, forma 120 combinações de curvas de retenção com oito pontos; por exemplo, a combinação B60.84 com 101 forma o seguinte conjunto de oito pontos de tensão: 1, 3, 6, 10, 35, 60, 84 e 101 kPa. (5)B60.84.101: conjunto base das tensões de 1, 3, 6, 10, 35, 60, 84 e 101 kPa, que, associado aos valores de tensão subseqüentes de 201 a $1515 \mathrm{kPa}$, forma 210 combinações de curvas de retenção com nove pontos; por exemplo, a combinação de B60.84.101 com 201 forma o seguinte conjunto de nove pontos de tensão: 1, 3, 6, 10, 35, 60, 84, 101 e 201 kPa. 
$\theta_{\mathrm{r}}$ ser igual ao teor de água em equilíbrio com a tensão de $1.515 \mathrm{kPa}\left(\theta_{1515}\right)$, conforme indicado por Assouline et al. (1998). Nesse processo de otimização, os resultados de $\theta_{\mathrm{r}}$ ficaram relativamente próximos de $\theta_{1515}$, os quais, analisados em termos da relação $\theta_{\mathrm{r}} / \theta_{1515}$, resultaram nos seguintes valores: 1,09 para o solo $\mathrm{RQ}_{1} ; 1,04$ para o $\mathrm{RQ}_{2}$; 0,92 para o $\mathrm{LVAd}_{1}$; 0,91 para o $\mathrm{LVAd}_{2}$ e 0,80 para o $\mathrm{LVd}$, mostrando que o valor de $\theta_{\mathrm{r}}$ que mais se distanciou do $\theta_{1515}$ foi o do solo $\mathrm{LVd}$, o qual foi cerca de $20 \%$ menor.

Os cinco pontos iniciais de tensão (1, 3, 6, 10 e $35 \mathrm{kPa}$ ), adotados como básicos na formação das curvas de retenção com um menor número de pontos, se localizam na porção da curva onde está presente a maior quantidade da água no solo entre os limites de $\theta_{\mathrm{s}}$ e $\theta_{\mathrm{r}}$ (Figura 1). A escolha desses pontos de tensão está de acordo com Silva et al. (1990), exceto pelo fato de que eles escolheram 5 e $20 \mathrm{kPa}$ no lugar de 6 e $35 \mathrm{kPa}$, os quais são usualmente associados aos conteúdos de água na microporosidade e na capacidade de campo (Embrapa, 1997). Neste trabalho, a quantidade de água contida no solo entre $\theta_{\mathrm{s}}$ e $\theta_{35 \mathrm{kPa}}$, em relação à diferença $\theta_{\mathrm{s}}-\theta_{\mathrm{r}}$, foi de $79,3 \%$ no solo $\mathrm{RQ}_{1}$, de $80,6 \%$ no $\mathrm{RQ}_{2}$, de $65,8 \%$ no $\operatorname{LVAd}_{1}$, de $62,48 \%$ no $\operatorname{LVAd}_{2}$ e de $55,0 \%$ no LVd. Além disso, ao observar as diferenças no valor de $\theta_{\mathrm{r}}$ entre as curvas de retenção de água, verifica-se que existe tendência de aumento na capacidade de retenção de água em $1.515 \mathrm{kPa}$ com o incremento no teor de argila do solo, conforme indicado por Ritchie et al. (1999).

Resultados da avaliação da soma de quadrados das diferenças ( $S Q_{\text {erro }}$ ) entre os conteúdos de água medidos no levantamento da curva de retenção, com 15 pontos e os respectivos valores calculados, usando o modelo de Genuchten (1980), para cada uma das 385 alternativas de curva de retenção para cada solo, foram analisados em termos de sua freqüência de ocorrência. Essa análise revelou ser a distribuição desses erros log-normal, ou seja, a transformação dos valores de SQerro pelo logaritmo neperiano Ln (SQerro) torna a distribuição normalmente distribuída (Neufeld, 2003). Essa distribuição foi padronizada utilizando as respectivas médias e o desvio-padrão de cada conjunto de 385 valores de Ln (SQerro), para possibilitar sua comparação entre os diferentes solos. Na representação padronizada estão apresentadas as freqüências acumuladas decorrentes dos 1.925 valores de $\operatorname{Ln}\left(\mathrm{SQ}_{\text {erro}}\right)$, que foram distribuídos em nove classes (Iman \& Conover, 1983), para cada solo (Figura 2). A alta correlação, de 0,9976, verificada entre a freqüência acumulada dos valores de Ln (SQerro) e a correspondente distribuição normal teórica demonstra o elevado grau de ajuste dos dados ao modelo de distribuição utilizado, o qual, de acordo com o teste r de Pearson (Levin, 1987), é de praticamente $100 \%$ de significância.

Por meio do modelo de distribuição (Figura 2) foi possível calcular a probabilidade acumulada de ocorrência do valor de Ln (SQerro) correspondente a cada uma das 385 curvas de retenção de água, para cada solo. Assim, para uma mesma combinação de pontos de tensão, pode-se chegar a um valor médio de probabilidade de ocorrência da curva de retenção correspondente ao conjunto dos cinco solos analisados. Esse valor médio de probabilidade foi utilizado como critério de classificação em ordem crescente das 385 combinações de curva de retenção. Em cada grupo de curvas de retenção com seis, sete, oito e nove pontos de tensão, foi selecionada a combinação que resultou no menor valor de probabilidade acumulada de ocorrência.

$\mathrm{O}$ aumento no número de pontos torna a curva de retenção mais próxima daquela considerada como referência (Tabela 4). Por exemplo, o melhor caso, cor-

Tabela 3. Parâmetros de ajuste das curvas de retenção de água, segundo o modelo de Genutchen (1980), para diferentes solos, determinados pelo procedimento de regressão não linear, utilizando 15 pontos de tensão(1).

\begin{tabular}{lccccc}
\hline Parâmetro & \multicolumn{5}{c}{ Classe de solo } \\
\cline { 2 - 5 } & $\mathrm{RQ}_{1}$ & $\mathrm{RQ}_{2}$ & $\mathrm{LVAd}_{1}$ & $\mathrm{LVAd}_{2}$ & $\mathrm{LVd}$ \\
\hline$\theta_{\mathrm{s}}\left(\mathrm{cm}^{3} \mathrm{~cm}^{-3}\right)$ & 0,4436 & 0,4617 & 0,4532 & 0,5163 & 0,4797 \\
$\theta_{\mathrm{r}}\left(\mathrm{cm}^{3} \mathrm{~cm}^{-3}\right)$ & 0,0889 & 0,1265 & 0,1630 & 0,2474 & 0,1861 \\
$\mathrm{n}$ & 1,9057 & 1,9263 & 1,4469 & 1,3690 & 1,2847 \\
$\alpha\left(\mathrm{kPa}^{-1}\right)$ & 0,4012 & 0,3556 & 0,3462 & 0,3821 & 0,3111 \\
\hline
\end{tabular}

${ }^{(1)}$ Modelo de Genutchen (1980): $\theta=\theta_{\mathrm{r}}+\left(\theta_{\mathrm{s}}-\theta_{\mathrm{r}}\right)\left\{1+(\alpha \mathrm{h})^{\mathrm{n}}\right\}^{(-1+1 / \mathrm{n})}$, em que $\theta$ é o conteúdo volumétrico da água no solo $\left(\mathrm{cm}^{3} \mathrm{~cm}^{-3}\right) ; \theta_{\mathrm{r}}$ é o conteúdo volumétrico residual da água no solo $\left(\mathrm{cm}^{3} \mathrm{~cm}^{-3}\right) ; \theta_{\mathrm{s}}$ é o conteúdo volumétrico saturado da água no solo $\left(\mathrm{cm}^{3} \mathrm{~cm}^{-3}\right)$; $\mathrm{n}$ é o fator de ajuste $($ sem dimensão); $\alpha$ é o fator de ajuste $\left(\mathrm{kPa}^{-1}\right)$. 
respondente à curva de retenção com nove pontos de tensão, resultou na probabilidade de ocorrência média de 0,0797; seguida da curva de oito pontos, com 0,1317 ; a de sete pontos, com 0,3537; e, finalmente, a de seis pontos, com 0,6177. Essa medida de probabilidade indica a chance de se obter uma combinação de pontos de tensão que resulte em uma curva de retenção melhor do que a indicada. Por exemplo, a probabilidade de ocorrência de 0,0797 indica que existe somente $7,97 \%$ de probabilidade de, pelo acaso, ser encontrada uma combinação qualquer de pontos de tensão melhor do que a curva de retenção formada pelos pontos de tensão: 1, 3, 6, 10, 35, $84,406,1.027$ e $1.515 \mathrm{kPa}$. No caso da curva de retenção de seis pontos, a probabilidade acumulada de 0,6177 indica que existe cerca de $61,77 \%$ de chance

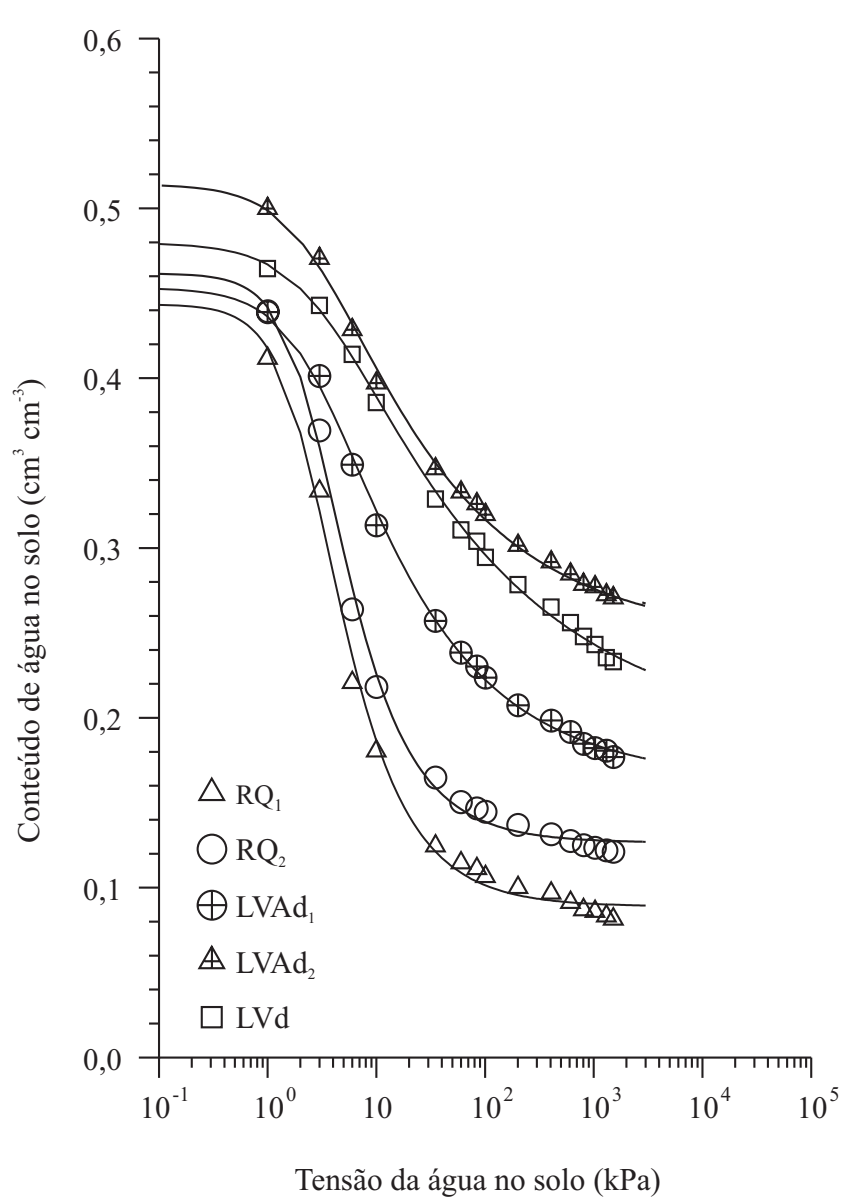

Figura 1. Curvas de retenção de água de solos do Cerrado, determinadas com 15 pontos de tensão e ajustadas ao modelo de Genutchen (1980). de ser encontrada, ao acaso, uma curva de retenção mais representativa do que aquela determinada pela combinação dos pontos de tensão de 1, 3, 5, 10, 35 e $101 \mathrm{kPa}$, tornando essa alternativa a menos confiável. A melhor das alternativas de curva de retenção de seis pontos aparece na posição de ordem 285, de um total de 385 alternativas.

Na análise de comparação de médias das probabilidades de ocorrência associadas às combinações de pontos de tensão (Tabela 4), foi possível identificar o grau de significância das diferenças apresentadas, usando o teste t unilateral (Neufeld, 2003). Ao se comparar as médias de probabilidade correspondentes às combinações de tensão de nove e oito pontos, verificou-se que existe a probabilidade de $8,5 \%$, ou menos, da hipótese de igualdade entre essas médias não ser verdadeira. Admitindo

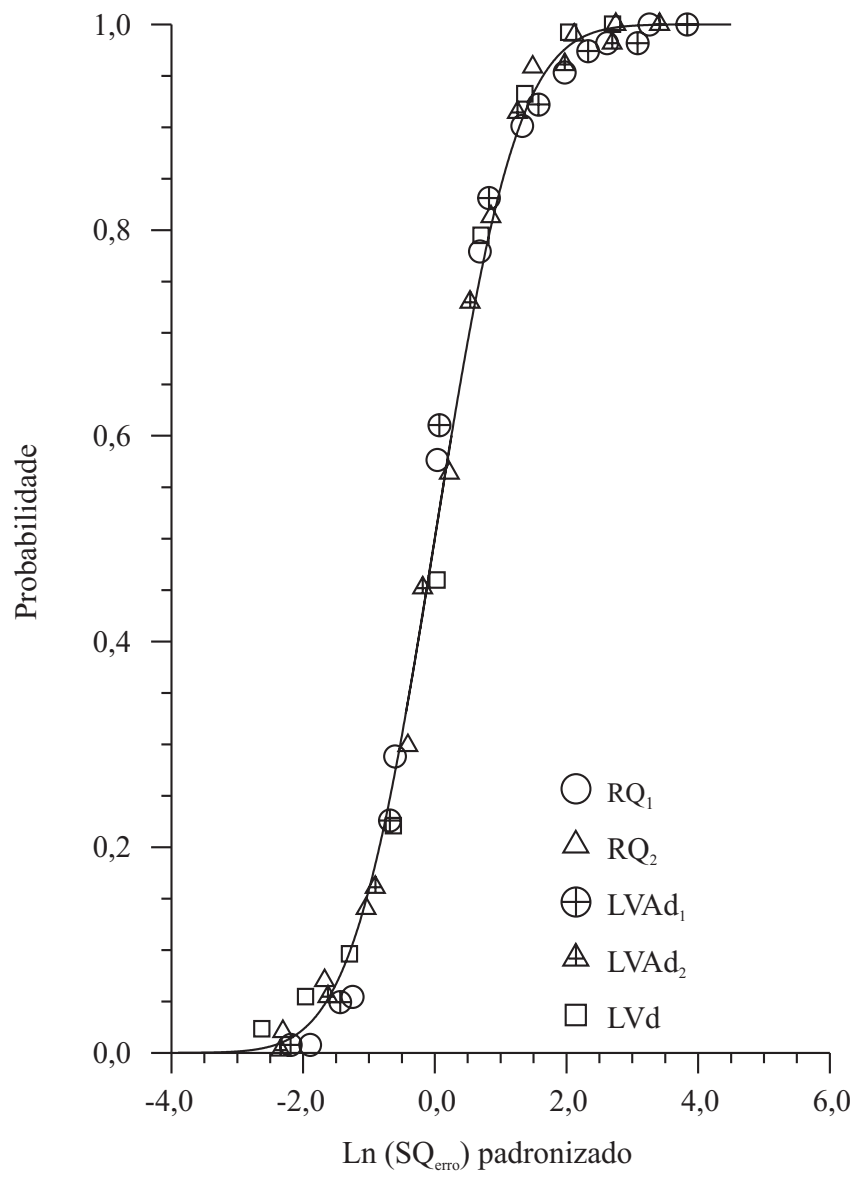

Figura 2. Comparação entre a freqüência acumulada (pontos) dos valores padronizados de $\operatorname{Ln}\left(\mathrm{SQ}_{\text {erro }}\right)$ e a probabilidade acumulada (linha) calculada pela distribuição Ln Normal para os diferentes solos analisados. 
Tabela 4. Probabilidade de ocorrência de uma combinação qualquer de pontos de tensão melhor do que a indicada.

\begin{tabular}{lcccccccc}
\hline Melhores combinações & \multirow{2}{*}{$\begin{array}{c}\text { Ordem de } \\
\text { de pontos }\end{array}$} & \multirow{2}{*}{$\begin{array}{c}\text { Número de } \\
\text { classificação }\end{array}$} & pontos & & \multicolumn{5}{c}{ Classe de solo } \\
\cline { 4 - 9 } & 1 & 9 & 0,0992 & 0,1101 & 0,0671 & 0,1215 & 0,0008 & $0,0797 \mathrm{a}$ \\
\hline B84.406.1027.1515 & 12 & 8 & 0,1295 & 0,1798 & 0,1419 & 0,1755 & 0,0317 & $0,1317 \mathrm{a}$ \\
B84.611.1515 & 119 & 7 & 0,1491 & 0,4282 & 0,2897 & 0,2475 & 0,6542 & $0,3537 \mathrm{~b}$ \\
B84.1515 & 285 & 6 & 0,4443 & 0,3964 & 0,7404 & 0,7904 & 0,7133 & $0,6177 \mathrm{c}$ \\
B101 & & & & & & & &
\end{tabular}

(1)B: conjunto das tensões de 1, 3, 6, 10, e $35 \mathrm{kPa}$, que, em associação com as demais tensões subseqüentes, até $1.515 \mathrm{kPa}$, foi usado para formar a combinação indicada; por exemplo, a combinação B84.406.1027.1515 representa B em associação com as tensões de 84, 406, 1.027, 1.515 kPa.

${ }^{(2)}$ Valores seguidos da mesma letra não foram significativos a 5\% de probabilidade pelo teste t unilateral.

o valor crítico normalmente convencionado de 5,0\% (Benjamin \& Cornell, 1970), para rejeição da hipótese de igualdade entre as médias, então, concluiu-se que as duas combinações de pontos de tensão testadas (oito e nove) são significativamente iguais, na probabilidade de $8,5 \%$, de modo que a opção de oito pontos torna-se mais vantajosa, por consumir menos tempo no levantamento da curva de retenção. Essa mesma análise, aplicada na comparação entre as combinações de sete e oito pontos, revelou a probabilidade de somente $2,98 \%$, ou menos, de rejeição da hipótese de igualdade entre essas médias; abaixo, portanto, do nível crítico adotado de 5\%, levando à conclusão de que não seria recomendável utilizar a curva de retenção com sete pontos. Verificou-se, também, por esse mesmo raciocínio que a curva de retenção com seis pontos, com uma probabilidade média de ocorrência de 0,6177 e, portanto, maior do que a de sete pontos (0,3537), é estatisticamente mais discrepante do que a curva gerada com a combinação de oito pontos, não devendo ser utilizada para o levantamento da curva de retenção de água, em solos do Cerrado.

\section{Conclusão}

A combinação de oito pontos de tensão, definida pelos valores de 1, 3, 6, 10, 35, 84, 611 e $1.515 \mathrm{kPa}$, é a recomendada para o levantamento das curvas de retenção de água de solos do Cerrado.

\section{Agradecimentos}

Ao assistente de pesquisa Wantuir Caixeta, da Embrapa Cerrados, pelas análises laboratoriais; ao Macroprograma II da Embrapa, pelo suporte financeiro ao projeto de pesquisa; ao CNPq, pela concessão de bolsa PROFIX ao quarto autor; à pesquisadora Adriana Reatto, da Embrapa Cerrados, pela orientação na classificação dos solos.

\section{Referências}

ABREU, S.L.; REICHERT, J.M.; REINERT, D.J. Escarificação mecânica e biológica para a redução da compactação em argissolo franco-arenoso sob plantio direto. Revista Brasileira de Ciência do Solo, v.28, p.519-531, 2004.

ASSOULINE, S.; TESSIER, D.; BRUAND, A. A conceptual model of the soil water retention curve. Water Resources Research, v.34, p.223-231, 1998.

BENJAMIN, J.R.; CORNELL, C.A. Probability, statistics, and decision for engineers. New York: McGraw-Hill, 1970. 684p.

BROOKS, R.H.; COREY, A.T. Properties of porous media affecting fluid flow. Journal of Irrigation and Drainage Division, v.92, p.61-88, 1966.

CINTRA, F.L.D.; PORTELA, J.C.; NOGUEIRA, L.C. Caracterização física e hídrica em solos dos Tabuleiros Costeiros no distrito de irrigação Platô de Neópolis. Revista Brasileira de Engenharia Agrícola e Ambiental, v.8, p.45-50, 2004.

EMBRAPA. Centro Nacional de Pesquisa de Solos (Rio de Janeiro, RJ). Manual de métodos de análise de solos. 2.ed. Rio de Janeiro, 1997. 212p.

GENUCHTEN, M.T. van. A closed-form equation for predicting the hydraulic conductivity of unsaturated soils. Soil Science Society of America Journal, v.44, p.892-898, 1980.

IMAN, R.L.; CONOVER, W.J. A modern approach to statistics. New York: J. Wiley, 1983. 497p.

KASTANEK, F.J.; NIELSEN, D.R. Description of soil water characteristics using cubic spline interpolation. Soil Science Society of America Journal, v.65, p.279-283, 2001.

LEVIN, J. Estatística aplicada a ciências humanas. 2.ed. São Paulo: Harbra, 1987. 392p.

MACEDO, J.R. de; OTTONI FILHO, T.B.; MENEGUELLI, N. doA. Variabilidade de características, físicas, químicas e físico-hídricas 
em solo Podzólico Vermelho-Amarelo de Seropédica, RJ. Pesquisa Agropecuária Brasileira, v.33, p.2043-2053, 1998.

NEUFELD, J.L. Estatística aplicada à administração usando Excel. São Paulo: Prentice Hall, 2003. 434p.

PRUNTY, L.; CASEY, F.X.M. Soil water retention curve description using a flexible smooth function. Vadose Zone Journal, v.1, p.179185, 2002.

REATTO, A.; CORREIA, J.R.; SPERA, S.T. Solos do bioma Cerrado: aspectos pedológicos. In: SANO, S.M.; ALMEIDA, S.P. de (Ed.). Cerrado: ambiente e flora. Planaltina: Embrapa-CPAC, 1998. p.47-86.

RICHARDS, L.A. Physical conditions of water in soil. In: BLACK, C.A.; EVANS, D.D.; WHITE, J.L.; ENSMINGER, L.E.; CLARK, F.E. (Ed.). Methods of soil analysis: physical and mineralogical properties, including statistics of measurements and sampling. Madison: American Society of Agronomy, 1965. p.128-152.

RITCHIE, J.T.; GERAKIS, A.; SULEIMAN, A. Simple model to estimate field-measured soil water limits. Transactions of the ASAE, v.42, p.1609-1614, 1999.
ROSSI, C.; NIMMO, J.R. Modeling of soil water retention from saturation to oven dryness. Water Resources Research, v.30, p.701708, 1994.

SILVA, A. de S.S.; BRITO, L.T. de L.; OLIVEIRA, C.A.V. de; MOITA, A.W. Parâmetros de solo em função da umidade na capacidade de campo em áreas irrigáveis do trópico semi-árido brasileiro. Pesquisa Agropecuária Brasileira, v.25, p.103-116, 1990.

SILVA, E.M. da; AZEVEDO, J.A. de. Influência do período de centrifugação na curva de retenção de água em solos de Cerrado. Pesquisa Agropecuária Brasileira, v.37, p.1487-1494, 2002.

SILVA, E.M. da; AZEVEDO, J.A. de. Período de centrifugação adequado para levantamento da curva de retenção da água em solos do bioma Cerrado. Planaltina: Embrapa Cerrados, 2001. 40p. (Boletim de pesquisa e desenvolvimento, 8).

SPERA, S.T.; REATTO, A.; CORREIA, J.R.; SILVA, J.C.S. Características físicas de um Latossolo Vermelho-Escuro no cerrado de Planaltina, DF, submetido à ação do fogo. Pesquisa Agropecuária Brasileira, v.35, p.1817-1824, 2000.

Recebido em 10 de dezembro de 2004 e aprovado em 13 de junho de 2005 